\title{
LTE Radio Measurements Above Urban Rooftops for Aerial Communications
}

\author{
Rafhael Amorim*, Huan Nguyen*, Jeroen Wigard ${ }^{\dagger}$, István Z. Kovács ${ }^{\dagger}$, Troels B. Sorensen*, Preben Mogensen*†, \\ *Department of Electronic Systems, Aalborg University, Denmark \\ $\{$ rma, hon, tbs, pm\}@es.aau.dk \\ ${ }^{\dagger}$ Nokia Bell Labs, Aalborg, Denmark \\ \{jeroen.wigard, istvan.kovacs, preben.mogensen\}@nokia-bell-labs.com
}

\begin{abstract}
This paper focus on the investigation of aerial communications targeting drones flying above rooftops in urban scenarios in a near future. A radio scanner is attached to a construction-lift to measure the radio signal from three different live LTE networks $(800,1800$, and $2600 \mathrm{MHz})$ at heights up to $40 \mathrm{~m}$ in urban senarios. The measurements suggest that the clearance of the radio path cause the number of cells in the detectable range of the equipment to increase. Also, it is found more neighbors within $3 \mathrm{~dB}$ of the serving cell in the receiver. There are 4 or more neighbors in this range in $2.6 \%, 4.2 \%$ and $7.4 \%$ of the samples in each frequency respectively at $100 \mathrm{~m}$, while this number does not exceed $1 \%$ at $1.5 \mathrm{~m}$. The paper also compares the path losses observed during the measurements with 3GPP reference models and previous studies. At last, heightdependent closed form expressions for the path loss slope and shadowing variation are provided for future investigations under similar scenarios.
\end{abstract}

\section{INTRODUCTION}

In recent years, an impressive growth on Unmanned Aerial Vehicles' (UAVs) market has been observed. This increase and the steady technological development of these devices are expected to enable a large number of new applications. It represents a market opportunity for cellular operators: UAVs will require data link connections, either for telemetry, command and control exchanges or potentially for real-time applications. In the first example, the data link must be highly-reliable due to safety concerns, in the latter it can demand high capacity, e.g. for real-time footage streaming.

Cellular networks engineers have years of experience in planning and optimizing their infrastructure for ground coverage using prediction tools and simulators. But the channel models currently used for this task do not extend for heights above rooftops, the region where most airborne UAVs (a.k.a. drones) are likely to be found. For this reason, it is important to model the propagation channel between cellular base stations (BSs) and airborne UAVs, to be used as an input for the assessment of this data link performance.

Lately, some efforts have been driven to this task. At the time of writing, the 3rd Generation Partnership Project (3GPP) holds a study item on enhanced support for aerial vehicles [1], which includes discussions on pathloss models and scenario definition to be used for simulation purposes.

Some previous works have addressed the link between BS and mobile terminals attached to UAVs. Matolak and Sun have contributed with an extensive set of studies that evaluates measurements in different scenarios in [2][3][4][5]. In these studies, measurements were performed for large distances in dedicated links in $\mathrm{C}$ and $\mathrm{L}$ bands, and are focused on UAV flying heights between $500 \mathrm{~m}$ and $2 \mathrm{~km}$. In [4], the path loss slope observed in measurements collected on suburban/near urban scenarios is on the range of 1.5 to 2.0 .

In other previous work, an indication of clearance in the radio path with higher UE heights is observed in [6], where authors report reduced shadowing variation, increased intersite interference power and higher number of visible neighboring cells. In [7] a modified two-ray channel model was presented, introducing variation in path loss exponents according to the UE height, based on GSM and UMTS measurements collected by a stationary balloon.

This paper aims to provide propagation models for UAVs connected to cellular networks. Measurements are currently focused at low heights $(\leq 150 \mathrm{~m})$, where we assume some of future drones applications and services will be deployed, such as infrastructure maintenance, surveillance and last mile packet deliveries [8]. In a previous study, measurements were collected from two LTE operators at the $800 \mathrm{MHz}$ band in a rural scenario with a scanner attached to a commercial UAV flying in heights up to $120 \mathrm{~m}$ [9]. The results showed radio path clearance as the UAV moves up: reduced shadowing variation, larger set of detected neighbor cells and path loss slope close to the theoretical free space path (FSPL) loss model .

This work presents measurements collected in live LTE networks for 3 different frequencies $(800,1800$ and 2600 $\mathrm{MHz}$ ) in urban scenarios on Northern Denmark. Measured heights vary from ground level to heights above rooftops, up to $40 \mathrm{~m}$ (see Section II). the measurements were collected with the help of a construction lift. The learnings acquired with this setup will be used in the future in the designing of a setup for urban measurements with a real UAV. Among others, this paper analyses the height dependency of the path loss slope, and compare the observed values with those currently adopted by 3GPP RAN 1 study item.

This paper is organized as follows: Section II introduces the scenario, measurement setup and the data processing methodology used in this investigation. Results and conclusions are followed, respectively, in Sections III and IV. 


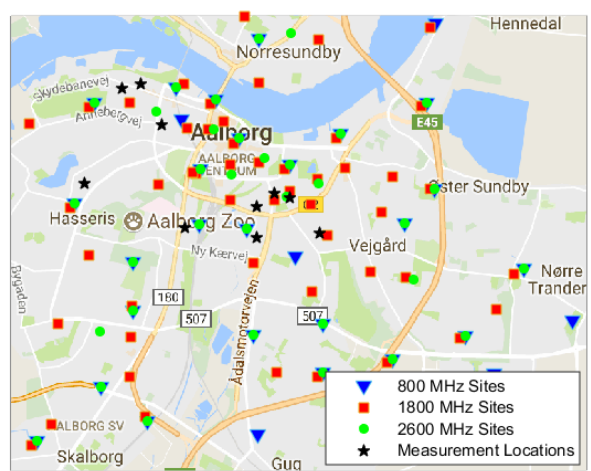

Fig. 1. Map with measurement and site locations

\section{Measurement Setup and Data Processing}

The measurement equipment used in the measurements is a $R \& S^{\circledR}{ }^{T S M A}{ }^{1}$ radio scanner. The device was set to scan three different frequencies from LTE live networks: 800, 1800 and $2600 \mathrm{MHz}$. Among the information saved on the measurement report are of particular interest for this paper: the device's GPS location and physical cell ID (PCI) and the average received power per LTE resource element (RE) [10] on the synchronization channel from serving and neighboring cells. The number of reported neighboring cells on each sample depends on the capability of the scanner of rightfully decode cell's synchronization channel. The signal-to-noise ratio (SINR) threshold values observed for this detection in the measurements is around $-20 \mathrm{~dB}$. The observed sampling rate in all trials was in the range of 3.4 and $7.5 \mathrm{~Hz}$.

With the purpose of measuring the propagation channel at different heights, the scanner was mounted together with an omni-directional antenna on a $1.8 \mathrm{~m}$-height mast inside a construction lift's basket, and then, lifted from 5 to $40 \mathrm{~m}$ height, with incremental steps of $5 \mathrm{~m}$. The basket was kept for 3 minutes on each of these levels, and lateral movements of 3-5m were induced within this period, aiming at mitigate eventual bias caused by small scale fading. Due to limitations on the lift's mobility, this procedure was repeated on 10 different locations, to introduce sampling variability (see Fig.1). Additionally, reference measurements were collected on ground level, with the mounting carried by pedestrian users around each location.

The measurements took place in the city of Aalborg, in Northern Denmark. For reference of scenario's characterization, the urban population in the city is just above 110,000 people $^{2}$ with a populational density of $\approx 1000$ inhabitants per $\mathrm{km}^{2}$. More detailed information on the chosen locations is found on Table I, such as the average and $90 \%$-ile of building heights in a $50 \mathrm{~m}$ radius around the measurement spot (showed in Fig. 1). Table II shows general information for the three

\footnotetext{
${ }^{1}$ https://www.rohde-schwarz.com/dk/brochure-datasheet/tsma/

${ }^{2} \mathrm{http}: / /$ denmark.dk/en/quick-facts/facts
}

networks, such as inter-site distance (ISD), mean transmitter heights and average downtilt in degrees, in the city center, within a radius of $4 \mathrm{~km}$.

TABLE I

LOCATIONS DESCRIPTION

\begin{tabular}{|c|c|c|c|}
\hline \multirow{2}{*}{ \# of Locations } & \multicolumn{2}{|c|}{ Building Heights } & \\
& Avg. (m) & $90 \%$-ile $(\mathrm{m})$ & Description \\
\hline 4 & $3.6-6.9$ & $4.4-9.8$ & Low Residential Area \\
\hline 4 & $11.8-15.5$ & $14.2-15.9$ & High Residential Area \\
\hline 2 & $4.4-5.1$ & $5.8-7.1$ & Low Industrial Area \\
\hline
\end{tabular}

TABLE II

NETWORKS GENERAL INFORMAITON

\begin{tabular}{|c|c|c|c|}
\hline $\begin{array}{c}\text { Frequency } \\
(\mathrm{MHz})\end{array}$ & $\begin{array}{c}\text { ISD } \\
(\mathrm{m})\end{array}$ & $\begin{array}{c}\text { Avg. } \\
\text { Tx. Height }(\mathrm{m})\end{array}$ & $\begin{array}{c}\text { Avg. Downtilt } \\
\text { (degrees) }\end{array}$ \\
\hline 800 & 850 & 26.2 & 5.5 \\
\hline 1800 & 580 & 26.3 & 5.3 \\
\hline 2600 & 690 & 25.3 & 5.8 \\
\hline
\end{tabular}

The data processing is similar to that previously used and detailed in [9]. Summarized here for the convenience of the reader: the PCI information was used to map the BS configuration, such as transmitted power, antenna used and site location. Pathloss samples were obtained by subtracting the measured power level from serving and neighboring cells from the EIRP (effective isotropic radiated power) of each base station. For the EIRP calculation the antenna gain used is calculated through the horizontal interpolation algorithm (HPI) applied over the horizontal and vertical antenna diagrams. Fast fading components are removed by applying local average on the path loss samples over windows of 40 wavelengths [11]. In order to avoid the roll-off region of the antenna patterns samples outside the $-12 \mathrm{~dB}$ vertical and horizontal lobes were filtered out from the analysis. In [9], the threshold value was $-6 \mathrm{~dB}$, but it had to be relaxed for the purpose of this study, as it deals with much closer ranges to the base stations, to avoid all samples at the 2 highest lift levels would be excluded from the analysis due to the steep elevation angles.

The outcome of the processing phase was then used to obtain coefficients to fit a generalization of the close-in lognormal path loss model [12]:

$$
P L(d, f, h)=\alpha(h) 10 \log _{10}(d)+P L_{r e f}(f)+X_{\sigma}(h) \quad[d B]
$$

where the variables $d, f$ and $h$ denote, respectively, the distance between BS and UAV (in $\mathrm{m}$ ), the center frequency $(\mathrm{MHz})$ and the UAV height above ground level $(\mathrm{m})$. Besides, $\alpha(h)$ represents the height-dependent path loss slope and $X_{\sigma}(h)$ is a Gaussian variable with zero mean and standard deviation $\sigma(h)$ that accounts for the shadowing variation at the height $h$. Finally, $P L_{r e f}$ stands for the close-in path loss reference at $1 \mathrm{~m}$ distance - assumed to be the theoretical free space path $\operatorname{loss}\left(P L_{r e f}=20 \log _{10}(f)+20 \log _{10}(4 \pi)\right)$.

\section{A. 3GPP Reference Model}

The working assumptions, at the time of writing, in the 3GPP study item on enhanced support for UAVs [1] [13] 
will be used for comparison purposes. Such a model is an extension of the well-known 3GPP model for the propagation channel for heights below $22.5 \mathrm{~m}$ that can be found in [14]. The measurement scenario differs from the assumptions of 3 GPP in ISD (500m) and antenna downtilt (10 degrees). For the convenience of the reader the model is repeated here in set of equations 2 and 3 for the line-of-sight (LOS) and non-lineof-sight (NLOS) cases, $P L_{L O S}^{\prime}$ and $P L_{N L O S}^{\prime}$, respectively.

$$
\begin{aligned}
& P L_{L O S}^{\prime}(d)=\left\{\begin{array}{l}
P L_{1}(d) \quad \text { if } d_{2 d} \leq d_{b p} \\
P L_{2}(d) \quad \text { if } d_{2 d}>d_{b p}
\end{array}\right. \text { where } \\
& P L_{1}(d)=28+22 \log _{10}(d)+20 \log _{10}\left(f_{c}\right) \\
& P L_{2}(d)=28+40 \log _{10}(d)+20 \log _{10}\left(f_{c}\right)-9 \log _{10}\left(d_{b p}^{2}+\Delta_{h}^{2}\right) \\
& P L_{N L O S}^{\prime}=\left\{\begin{array}{l}
\max \left(P L_{L O S}^{\prime}(d), P L_{3}(d)\right) \quad \text { if } h_{u e} \leq 22.5 \\
P L_{4}(d) \quad \text { if } h_{u e}>22.5, \quad \text { where }
\end{array}\right. \\
& P L_{3}(d)=13.54-39.08 \log _{10}(d)+20 \log _{(}\left(f_{c}\right)-0.6\left(h_{u e}-1.5\right) \\
& P L_{4}(d)=22.5+\left(46-7 \log _{10}\left(h_{u e}\right)\right) \log _{10}(d)+20 \log _{10}\left(f_{c}\right)
\end{aligned}
$$

In these two set of equations, $d$ stands for the total 3D distance between the BS and the UE, while $d_{2 d}$ represents the 2D projected distance regardless the difference in heights, $\Delta_{h}$, between them. Also, $d_{b p}$ is a breakpoint distance as calculated in [14] and depends on both, the center frequency $f_{c}$ used for transmission and the UE heights $h_{u e}$. It is worthy noting that for UE heights, above $22.5 \mathrm{~m}$ there is no breakpoint distance for $P L_{L O S}$ to be considered within the range supported by the model $(5 \mathrm{~km})$.

\section{RESULTS}

The periodicity of saved measurement reports are internally controlled by the device based on multiple factors and cannot be directly controlled by the user. The recorded sampling rate observed for each height is showed in Table III.

TABLE III

OBSERVED SAMPLING RATES

\begin{tabular}{|c|c|c|c|}
\hline Height (m) & $\begin{array}{c}\text { Sampling } \\
\text { Rate (Hz) }\end{array}$ & Height (m) & $\begin{array}{c}\text { Sampling } \\
\text { Rate (Hz) }\end{array}$ \\
\hline 1.5 & 3.7 & 25 & 4.8 \\
\hline 5 & 3.8 & 30 & 5.9 \\
\hline 10 & 3.8 & 35 & 6.0 \\
\hline 15 & 3.8 & 40 & 6.3 \\
\hline 20 & 4 & $* * *$ & $* * *$ \\
\hline
\end{tabular}

The work in [9] reported that the average number of cells detected increased with UE heights, due to the clearance in the radio path. The same behavior was observed during the urban measurements with the lift, as exposed in Fig. 2. For all 3 frequencies, the number of cells tends to increase for the highest measurement compared to the ground level reference. The increase is specially high at $1800 \mathrm{MHz}$, which is the more dense network (see Table II), from 7.1 to 12.3. An interesting behavior is observed at $2.6 \mathrm{GHz}$, where the number of detected cells first decreases as the lift was elevated form ground level

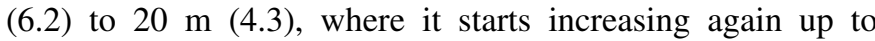
$40 \mathrm{~m}$ (8.9). A cell is only recognized by the scanner if the synchronization channel is successfully decoded. If SINR is too low for that specific cell, it cannot be reported by the

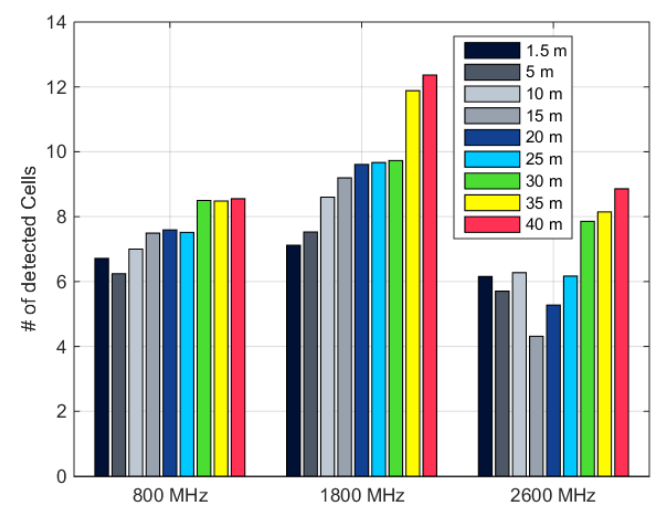

Fig. 2. Avg. Number of Detected cells per sample

scanner. In some cases, if the signals from few cells are very strong, other neighbor cells may not be detected even if their signal levels are not so weak, due to the interference observed in synchronization channels. For the $2.6 \mathrm{GHz}$, the clearance on the radio path of first few cells seems to have dominated at the first heights, increasing the interference levels on the sync channel and therefore reducing the overall number of detected cells. However, after $20 \mathrm{~m}$ of height, it seems that other cells further away also experience good radio clearance and their signals can overcome the SINR.

One can argue that power received from the strongest cell at higher heights could decrease, as the UE is moving away from the downtilted beams of the urban transmitter antennas. However, as showed in Fig. 3,the radio clearance is the dominant phenomenon up to $40 \mathrm{~m}$, as the strongest received power is around $20 \mathrm{~dB}$ higher at $40 \mathrm{~m}$ then at $1.5 \mathrm{~m}$. On the other hand, radio clearance also brings more interference concerns. When interfering signals are closer in power domain the interference cancellation mechanisms at the received end tends to perform worse. Fig. 4 shows the distribution of number of neighbor cells within $3 \mathrm{~dB}$ of the strongest (serving) cell signal. The number of neighbor cells lying in this power region tends to increase with UE heights. For instance, there are $2.6 \%$ $(800 \mathrm{MHz}), 4.2 \%(1800 \mathrm{MHz})$ and $7.4 \%(2600 \mathrm{MHz})$ samples with 4 or more sites within $3 \mathrm{~dB}$ of the serving cell at $40 \mathrm{~m}$ and less than $1 \%$ on ground level.

These two effects cited here can be even worse at higher heights. In [9] the average number of detected sites at $30 \mathrm{~m}$ is 7.6 and 16.9 at $120 \mathrm{~m}$; and in [15] it is showed that at $120 \mathrm{~m}$ it is necessary to cancel the interfernece of the 4 strongest neighbors to obtain $3 \mathrm{~dB}$ of SIR gain.

Regarding the path loss model obtained from the measurements, two examples are presented in Fig. 5 and 6, which show respectively the results observed at ground level and at $40 \mathrm{~m}$. The first consideration to be made regards the measured distances, which includes the range between $100 \mathrm{~m}$ and $5 \mathrm{~km}$ (similar across all trials). Hereafter, all path loss analysis are implicit referring to this range. For matters of comparison, 


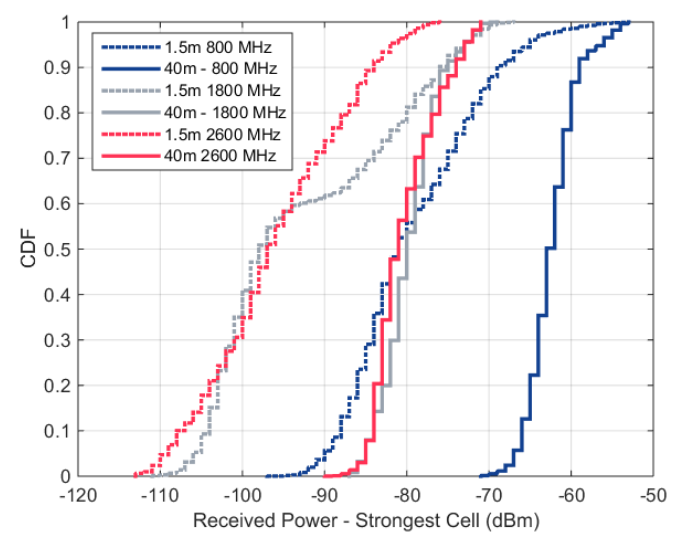

Fig. 3. CDF: Avg. Received power (dBm) for first cell
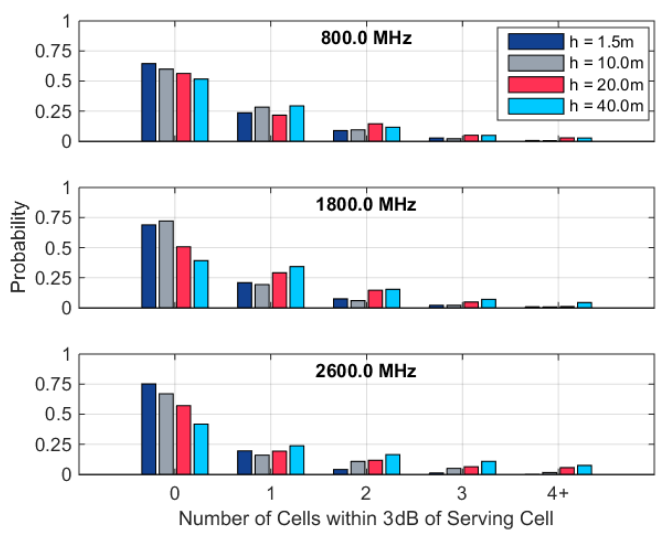

Fig. 4. Distribution of number of cells within $3 \mathrm{~dB}$ of the serving cell

the 3GPP model mentioned as reference is valid for distances between $10 \mathrm{~m}$ and $4 \mathrm{~km}$. In these plots all values in y-axis was subtracted from $P L_{r e f}$ eliminates the frequency dependent component of $20 \log _{10}(f)$ in eq.1, so they can all be showed together regardless the frequency.

In Fig. 5, at $1.5 \mathrm{~m}$, it is possible to see the losses are much above FSPL reference line. At distances around $1 \mathrm{~km}$, the excess losses (losses above FSPL reference) observed by the CI model line is about $30 \mathrm{~dB}$. Also, most data samples are above 3GPP LOS model, and it seems 3GPP NLOS for is a better approximation of the measured values. On the other hand, in Fig. 6, at $40 \mathrm{~m}$, the measurement results are much closer to FSPL, with excess losses of $5 \mathrm{~dB}$ at $1 \mathrm{~km}$; and much closer of 3GPP LOS model. It is worth noting that 3GPP NLOS model in this case is much more pessimistic than all recorded points, regardless the fact that current LOS probability model for such heights predicts values below $50 \%$ for this height [1].

A summary of the results is found in Table IV, with the values that fit eq. 1 . It is also added to this table $\bar{E}_{L O S}$ and $\bar{E}_{N L O S}$, which are the average deviations from 3GPP model and the recorded data samples for the LOS and NLOS models,

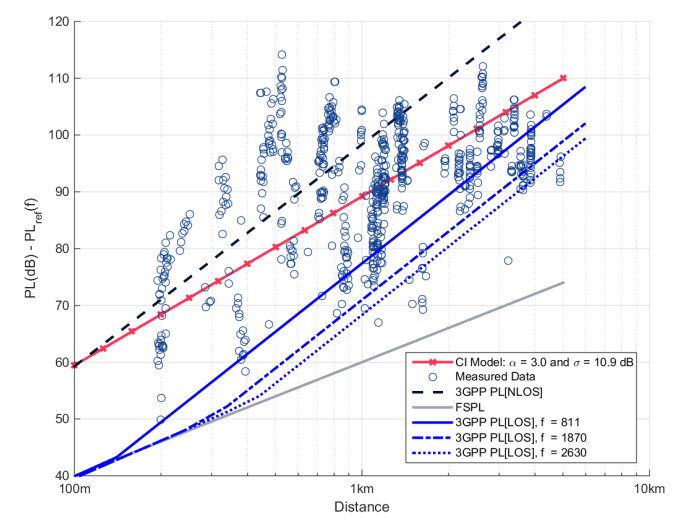

Fig. 5. Path Loss Models at $1.5 \mathrm{~m}$

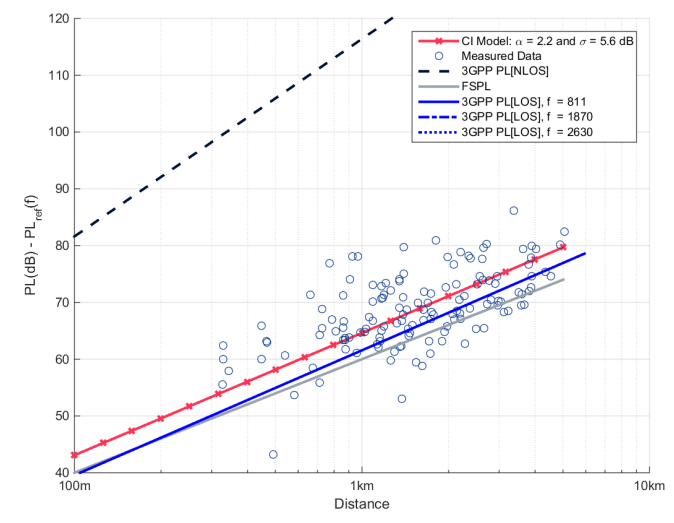

Fig. 6. Path Loss Models at 40m

respectively: positive values represent underestimation, while negative values are an overestimation. In each row, one of these two values is marked in bold to represent the one that better approximates (in absolute values) our measurements. It is worth noting that $\bar{E}_{L O S}$ is always positive, while $\bar{E}_{N L O S}$ is always negative. The 3GPP NLOS seems to present better estimation for heights up to $5 \mathrm{~m}$; while from $10 \mathrm{~m}$ onwards the LOS model is more representative, specially after $h_{u e}>25 \mathrm{~m}$ where it approximates FSPL behavior.

TABLE IV

AVERAGE DIFFERENCE FROM REFERENCE MODELS

\begin{tabular}{|c|c|c|c|c|}
\hline Height $(\mathrm{m})$ & $\alpha$ & $\sigma[\mathrm{dB}]$ & $\bar{E}_{L O S}$ & $\bar{E}_{N L O S}$ \\
\hline 1.5 & 3.0 & 10.9 & 18.2 & $\mathbf{- 9 . 0}$ \\
\hline 5 & 2.8 & 11 & 20.9 & $\mathbf{- 1 3 . 1}$ \\
\hline 10 & 2.5 & 12.4 & $\mathbf{1 3 . 5}$ & -18.4 \\
\hline 15 & 2.3 & 9.3 & $\mathbf{9 . 2}$ & -20.0 \\
\hline 20 & 2.2 & 8.2 & $\mathbf{5 . 2}$ & -21.9 \\
\hline 25 & 2.2 & 6.7 & $\mathbf{3 . 4}$ & -34.9 \\
\hline 30 & 2.2 & 6.0 & $\mathbf{3 . 6}$ & -33.4 \\
\hline 35 & 2.2 & 5.9 & $\mathbf{3 . 4}$ & -32.5 \\
\hline 40 & 2.2 & 5.6 & $\mathbf{3 . 1}$ & -32.7 \\
\hline
\end{tabular}



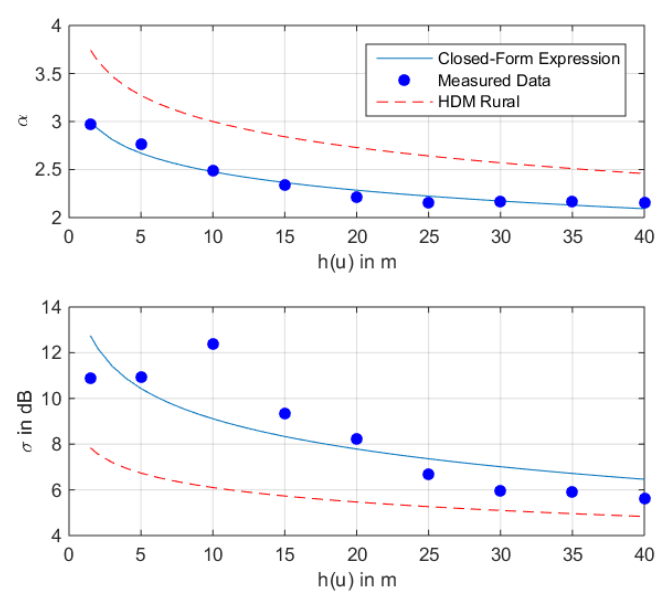

Fig. 7. Height Dependent Models for $\alpha$ and $\sigma$

The extended model used as of this writing, in [1] for $h_{u e}>$ $22.5 \mathrm{~m}$ seems to be over-pessimistic. The model changes so abruptly that $\bar{E}_{N L O S}$ changes from -21.9 to -34.9 , what cannot be attributed to changes in the measured data values, as showed by the values of $\alpha$ and $\bar{E}_{L O S}$.

Compared to previous works, Table IV also shows the path loss slope and shadowing variation to reduces with UE gains in height, except for an outlier of $\sigma$ when $h_{u e}=10 \mathrm{~m}$. Based on this, a logarithmic model was derived to create closed-form expressions for $\alpha(h)$ and $\sigma(h)$ in eq. 1 to be used in evaluation of scenarios to the one described in this paper. The closed form expressions are presented in equations 4 and 5 .

$$
\begin{gathered}
\alpha(h)=-0.64 \log _{10}(h)+3.12 \\
\sigma(h)=-4.40 \log _{10}(h)+13.51
\end{gathered}
$$

Fig. 7 shows the closed-form expressions plotted against the measured values in Table IV and the height dependent model (HDM) for the rural measurements in [9]. The results in this paper show slope values smaller than those in [9], and higher shadowing deviation. It is important to note, though, besides the different types of environments, in this paper the distances are within the range of $100 \mathrm{~m}$ to $5 \mathrm{~km}$, while the HDM Rural model was built using samples collected in a different range of distances, between 1.5 and $17 \mathrm{~km}$.

\section{CONCLUSION}

A set of measurements were performed in urban scenarios in heights up to $40 \mathrm{~m}$, to emulate radio performance of drones connected over cellular networks, in low-elevation flights and approximation and taking-off heights. Similar results were observed for three different frequencies $(800,1800$ and 2600 $\mathrm{MHz}$ ). Previous references have suggested increases in the number of detected cells at higher heights, but our findings suggest that at $40 \mathrm{~m}$ there already is an sensible increase in number of neighbors. Moreover, there is a substantial increase in the received power by neighbor sources, which will translate into heavier interference to be overcome by the BS-Drone link.

Path loss investigations also showed that above $25 \mathrm{~m}$, the propagation approximates the FSPL. When compared to reference values in 3GPP the measurements suggest that current work assumptions for $P L_{N L O S}$ are too pessimistic and a bad predictions to what is observed on field. A lso a height dependency is observed for the slope of the path loss line and also for the shadowing variation of the data samples. Closedform expressions are provided for height-dependent models investigations in similar scenarios.

Next steps include using a real UAV to perform measurements at higher levels (up to $120 \mathrm{~m}$ ), as a manner to investigate how these effects extend for such heights, and how the higher density of sites in urban areas impacts the interference analysis previously provided for rural scenarios.

\section{ACKNOWLEDGMENT}

This research has received funding from the SESAR Joint Undertaking under the European Union's Horizon 2020 research and innovation programme, grant agreement No 763601.

\section{REFERENCES}

[1] "Enhanced LTE support for aerial vehicles," 3GPP, Tech. Rep. TS 36.777 V0.1.0, August 2017.

[2] D. W. Matolak and R. Sun, "Air-ground channel characterization for unmanned aircraft systems - part i: Methods, measurements, and models for over-water settings," IEEE Transactions on Vehicular Technology, vol. 66, no. 1, pp. 26-44, Jan 2017.

[3] R. Sun and D. W. Matolak, "Air-ground channel characterization for unmanned aircraft systems part ii: Hilly and mountainous settings," IEEE Transactions on Vehicular Technology, vol. 66, no. 3, pp. 1913-1925, March 2017.

[4] D. W. Matolak and R. Sun, "Air-ground channel characterization for unmanned aircraft systems - part iii: The suburban and near-urban environments," IEEE Transactions on Vehicular Technology, vol. 66, no. 8, pp. 6607-6618, Aug 2017.

[5] R. Sun, D. W. Matolak, and W. Rayess, "Air-ground channel characterization for unmanned aircraft systems - part iv: Airframe shadowing," IEEE Transactions on Vehicular Technology, vol. PP, no. 99, pp. 1-1, 2017.

[6] B. V. D. Bergh, A. Chiumento, and S. Pollin, "Lte in the sky: trading off propagation benefits with interference costs for aerial nodes," IEEE Communications Magazine, vol. 54, no. 5, pp. 44-50, May 2016.

[7] N. Goddemeier, K. Daniel, and C. Wietfeld, "Role-based connectivity management with realistic air-to-ground channels for cooperative uavs," IEEE Journal on Selected Areas in Communications, vol. 30, no. 5, pp. 951-963, June 2012.

[8] K. Welch, "Evolving cellular technologies for safer drone operation," Qualcomm 5G White Paper and Presentations, Tech. Rep., October 2016.

[9] R. Amorim, H. Nguyen, P. Mogensen, I. Z. Kovács, J. Wigard, and T. B. Sørensen, "Radio channel modeling for uav communication over cellular networks," IEEE Wireless Communications Letters, vol. 6, no. 4, pp. 514-517, Aug 2017.

[10] "Evolved Universal Terrestrial Radio Access (E-UTRA); Physical Layer Measurements," 3GPP, Tech. Rep. TS 36.214 V8.7.0, September 2009.

[11] W. C. Y. Lee, "Estimate of local average power of a mobile radio signal," IEEE Transactions on Vehicular Technology, vol. 34, no. 1, pp. 22-27, Feb 1985.

[12] T. Rappaport, Wireless Communications: Principles and Practice, ser. Prentice Hall communications engineering and emerging technologies series. Prentice Hall PTR, 2002.

[13] Ericsson, "Summary of Email discussion [89-10] on remaining details of channel modelling," 3GPP TSG-RAN WG1 \#90, Tech. Rep. R11715084, August 2017.

[14] "Study on channel model for frequencies from 0.5 to $100 \mathrm{GHz}$," 3GPP, Tech. Rep. TS 38.901 V14.1.1, May 2017.

[15] I. Kovacs, R. Amorim, H. Nguyen, J. Wigard, and P. Mogensen, Interference analysis for UAV connectivity over LTE using aerial radio measurements. IEEE Vehicular Technology Society, 52017. 